\title{
Application of Intima Media Thickness Measurement and Omics in Intra- uterine Growth Restriction Disease
}

Donata Favretto1* Erich Cosmi ${ }^{2}$ and Silvia Visentin ${ }^{2}$

${ }^{1}$ Department of Cardiological, Thoracic and Vascular Sciences, University of Padova, Padova, Italy

${ }^{2}$ Department of Woman's and Child's Health, University of Padova, Padova, Italy

\begin{abstract}
Intrauterine growth restriction is defined as a fetus with an estimated fetal weight less than 10th percentile for gestational age and cardiovascular changes, usually detected by means of Doppler ultrasonography. Noncommunicable diseases (such as cardiovascular diseases -CVD- and diabetes) still represent the main cause of mortality and morbidity in the industrialized world. Various studies support the hypothesis, formulated by Barker, that an adverse intrauterine environ-ment results in physiological adaptations of the fetus, maximizing its immediate chances for survival, but with detri-mental effects in adulthood. Low birth weight caused by IUGR was recently known to be associated with increased rates of CVD, non-insulin dependent diabetes in adult life, and neuromotor development alteration.

The ultrasound-based measurement of fetal aorta intima media thickness (aIMT) represents an easy marker to investigate the pre-atherosclerotic changes.

Omics research holds great promise for discoveries in nutrition research, including profiles and characteristics of dietary and body proteins; metabolism of nutrients; functions of nutrients and other dietary factors in growth, reproduction, and health. The proteome and metabolome analysis are expected to play an important role in understanding pathophysiological molecular mechanisms and in solving major nutrition-associated problems in humans, such as IUGR and cardiovascular disease.

This review focuses the importance to identify a class of fetuses at risk of cardiovascular disease in utero, childhood and adult life, combining clinical and omics markers. It should be interesting to combine functional and structural information discovered to develop preventative and/or interventional therapeutic strategies.
\end{abstract}

Keywords: Intrauterine growth restriction; Intima media thickness; Proteomics; Metabolomics; Fetal programming

\section{Intrauterine Growth Restriction, Cardiovascular Remodelling and Fetal Programming}

Intrauterine growth restriction (IUGR) defined as a fetus with an estimated fetal weight (EFW) less than $10^{\text {th }}$ percentile for gestational age and cardiovascular changes, usually detected by means of Doppler ultrasonography, occurs in $5-10 \%$ of pregnancies. Several factors, including genetic (intrinsic fetal conditions) and environmental (maternal and external conditions), can lead to IUGR and to serious consequences, such as metabolic, neuroendocrine and cardiovascular adaptations, in early and later life [1]. Birth weight and gestational age at birth are the most important predictors of neonatal mortality and morbidity, being responsible for $50 \%$ of preterm and $20 \%$ of term perinatal deaths, considering birth weight an independent risk factor for these adverse outcomes [2]. There is still a debate in the literature about the definition of IUGR, and the use of customized fetal growth charts has been proposed, taking into account anthropomorphic variables of the parents [3]. The American College uses the terms IUGR interchangeably in order to identify fetuses with EFW by ultrasound scan $<10$ th percentile for gestational age [1], while they used small for gestational age fetuses (SGA) in order to exclusively identify newborns whose birth weight is $<10$ th percentile. However, RCOG considers an abdominal circumference less than $10 \%$ sufficient to suspect SGA [4]. Fetal umbilical artery (UA) Doppler has been proposed to distinguish IUGR due to placental insufficiency from SGA fetuses, characterizing a different class of risk [5-6]. IUGR presenting Doppler abnormalities are in fact associated with higher incidence of perinatal death, especially in preterm fetuses, perinatal and neonatal morbidity, fetal distress during labour, and higher rate of caesarean section [7]. The identification of placental dysfunction by Doppler evaluation demonstrates placental vascular abnormality. Specifically, as the villous vascular tree becomes compromised, elevated UA Doppler blood flow resistance rises progressively, reflecting the structural abnormality [8]. Moreover, Doppler evaluation of fetal cerebral and venous circulations has amplified the placenta understanding, providing evidence that placentabased IUGR manifests progressive cardiovascular signs heralding fetal acidemia and stillbirth [9]. At an early stage of placental compromise a reduced volume of blood to the placenta maintains a relatively normal cardiac output. A progressive low cardiac output however leads the fetus to a growth reduction and changes in impedance to flow in fetal arterial and venous circulation, attempting to achieve more efficient extraction of oxygen and nutrients [10]. A brain and heart-sparing effects with a reduced flow to less important organs such as muscles, bowel, and kidneys represent adaptive strategies that become pathologic when deprivation becomes extreme and fetal distress supervenes [2]. Higher intravascular pressure acting perpendicular to the vessel wall should

*Corresponding author: Donata Favretto, Department of Cardiologic, Thoracic and Vascular Sciences, University of Padova Via Falloppio 50, Padova, Italy, Tel: +39 049 8272224; Fax: +39 049 8218927; E-mail: donata.favretto@gmail.com

Received: October 27, 2014; Accepted: December 25, 2014; Published: January 01, 2015

Citation: Favretto D, Cosmi E, Visentin S (2015) Application of Intima Media Thickness Measurement and Omics in Intra-uterine Growth Restriction Disease. Cardiol Pharmacol 4: 126. doi:10.4172/2329-6607.1000126

Copyright: (c) 2015 Favretto D et al. This is an open-access article distributed under the terms of the Creative Commons Attribution License, which permits unrestricted use, distribution, and reproduction in any medium, provided the original author and source are credited. 
create a shear stress on the surface of the endothelium up or down regulating differentially endothelial genes, leading to cell proliferation, apoptosis, matrix degradation and synthesis [11]. The condition of hypoxia even may lead to cardiac hypertrophy and to the disruption in the normal pattern of cardiomyocyte development [12].

Moreover, IUGR disorder results in a reduction cells' number, in particular in nephron number, with an increased blood flow through each glomerulus and subsequent glomerulosclerosis [13].

An endothelial damage and an altered renal flow and development, with a reduced nephron endowment in utero could predispose to coronary heart disease, stroke and hypertension in later life. This association between low birth weight (LBW) and cardiovascular disease (CVD) in adulthood, the so-called "Barker's hypothesis" [14], postulated that when a damage occurs in a critical period this may give rise to a range of different physiological or morphological states, in line with the concept of developmental plasticity. Variability and plasticity of physiological and behavioral responses help the growing organism to adapt effectively to the uncertainty of later environmental conditions. This fetal response is characterized by insulin resistance, a preference to lay down fat, reduced skeletal mass and bone mineralization, low capillary density in many tissues, endothelial dysfunction, reduced nephron number and lowered negative feedback in the hypothalamicadrenal axis [2].

In 2004, the World Health Organization reported cardiovascular diseases/ischemic heart disease to be the leading cause of death worldwide and that cardiovascular deaths are envisaged to escalate to 23.4 million by the year 2030 [15]. At the moment there are no proven preventive or therapeutic strategies, although recent progresses in clinical research have tried to optimize the timing of delivery, finding the best compromise between a preterm birth and a growth in uterus in non-optimal condition (Figure 1).

\section{Endothelial Dysfunction and Evaluation}

The mechanisms that relate fetal programming to LBW and endothelial dysfunction in utero is still largely unknown and different explanations have been proposed [14]. The vascular endothelium is a physical barrier between the blood and the vessel wall structures, and it consists of approximately 1-6×1013 endothelial cells and accounts for

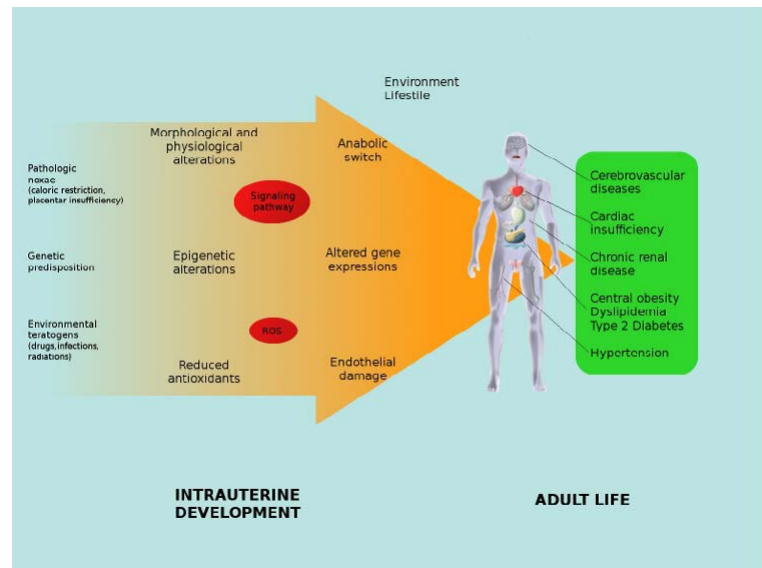

Figure 1: Programming factors of adult cardiovascular health. In presence of an unfavourable environment the fetus adapts by favouring growth of crucial organs such as the brain, the heart and the adrenal glands. The adaptation may cause structural, physiological and metabolic changes that influence the endothelial health later in life and the development of CVD in adult life. about $1 \mathrm{~kg}$ of total body weight. Vascular homeostasis entails keeping a tightly controlled balance between a vasodilatory state (i.e. nitric oxide (NO) and prostacyclin), which is often associated with anti-oxidant, anti-inflammatory and anti-thrombotic effects on one hand, and a vasoconstrictory state (i.e. thromboxane, free radicals, or endothelin) on the other, which is associated with pro-oxidant, pro-inflammatory and pro-thrombotic effects [16].

Cardiovascular risk factors or disorders such as hypertension, smoking, dyslipidaemia, diabetes mellitus, physical inactivity and obesity, are considered to be the top risk factors for cardiovascular mortality worldwide and may be involved in the development of endothelial dysfunction and atherosclerosis [16]. Several mechanisms may be involved in the development of endothelial dysfunction (ED), such as reduced synthesis and release of NO or enhanced inactivation of NO after its release from endothelial cells by radicals or oxidized low-density lipoprotein (LDL). Increased plasma levels of oxidized LDL, higher levels of cytokines and/or an activated renin-angiotensin system may be also involved the development of atherosclerotic disease [17]. Oxidative stress appears to be the common underlying cellular mechanism for the development of ED in all the risk factors discussed above. According to the literature, cardiovascular risk factors are associated with up-regulation of ROS sources, especially NADPH oxidase [18]. Harmful effects of oxidative stress include increasing vascular smooth muscle cells proliferation, resulting in thickening of the vascular wall, endothelial cell apoptosis, and increased expression and activity of matrix metalloproteinases, which are involved in the establishment of an atherosclerotic plaque. Superoxide anion is implicated in the direct induction of ED by the scavenging of NO, leading to the production of the highly reactive and harmful reactive nitrogen species, peroxynitrite [19]. High levels of peroxynitrite are injurious to the cells, oxidatively damaging DNA, lipids and proteins. In addition to being cytotoxic, peroxynitrite damages the intricate endothelial NOS (eNOS) structure, leading to eNOS uncoupling, which further perpetuates the ED vicious circle [20]. Inflammation is another common underlying mechanism of ED. Under physiological conditions, the endothelium regulates vascular inflammation (including expression of adhesion molecules and leukocyte adhesion) via the release of NO. It is therefore more likely that ED will promote sustained vascular inflammation, which is detrimental to the vascular system [21]. However, several studies have reported that inflammation also promotes ED and it is therefore recognised as a novel risk factor for cardiovascular diseases. Oxidative stress may amplify vascular inflammation signalling pathways, and conversely inflammatory cells increasingly release superoxide anion [22].

Inflammation is often associated with the overexpression of inflammatory cytokines such as tumour necrosis factor-alpha and interleukin-1. These inflammatory cytokines in turn prompt endothelial cells or macrophages to express adhesion molecules (VCAM-1, ICAM1, MCP-1, interleukin-6) resulting in a state of endothelial activation, which is a precursor of ED [23].

It's evident that the progression from ED to endothelial damage and atherosclerosis is multifactorial and that atherogenesis probably begins in early life. The examination of topographic distributions of atherosclerosis and their relation to risk factors for adult coronary heart disease in abdominal aortas of more than 2000 autopsied persons 15 through 34 years of age, reported in abdominal aortas of 15- to 24-year-old persons fatty streaks involving an elongated oval area on the dorsolateral intimal surface and another oval area in the middle third of the ventral surface. Raised lesions in 25- to 34-year-old persons involve an oval area in the distal third of the dorsolateral intimal surface. 
The observations reported in this study showed that risk factor effects, such as hemodynamic stresses related to arterial geometry, are greater in particular vulnerable regions of the aorta [24]. Moreover, a postmortem study performed on infants already showed that the abdominal aorta is the first site to be involved, indicating intimal thickening to be the earliest morphological aspect ${ }^{25}$. The abnormal findings observed in the aorta of fetuses and children consisted of proliferative intima thickness, fraying, splitting, and reduplication, disruption of the internal elastic membrane and microcystic degeneration of the inner media [25].

Recently, according to these studies, the improvement of imaging in health care allowed studying and analyzing the structure, thickness and functionality of several vessels.

An ideal method for the direct measurement of endothelial function should be safe, cost-effective, non-invasive, repeatable, and reproducible and standardized between laboratories. There are several methods of assessing endothelial function in adults include flow-mediated dilation, forearm plethysmography, finger-pulse plethysmography, pulse curve analysis, carotid intima media thickness and quantitative coronary angiography [26]. Ultrasound-based measurement of aorta intima media thickness (aIMT) in neonates and childhood has become a feasible, accurate, and sensitive marker of endothelial damage and cardiovascular risk [27]. Arterial compliance increases with age as arteries become larger during childhood growth and development, and may temper age-associated increases in arterial stiffness in young subjects, demonstrated by a increase of blood pressure (BP). The increased arterial diameters and/or large vessel elasticity that occur with growth and development may be adequate to compensate for increased arterial pressures. When tension in the aortic wall surpasses the point of natural adaptation, aortic stiffness would subsequently rise [28]. Experimental data highlighted that early atherosclerosis lesions are characterized by intima thickness due to the infiltration of smooth muscle cells, monocytes, and rare leukocytes, as well as interstitial glycosaminoglycan deposits [29]. Prenatal events leading to impaired fetal growth might be associated with structural changes in the aorta wall. The possibility of measuring both arterial diameters and wall thickness in fetuses by using a noninvasive technique such as ultrasound excited the interest in order to individuate early arterial wall lesions.

During fetal life, in the aortic wall the intima consists of a single layer of endothelial cells. The internal elastic membrane (IEM), continuous, fenestrated, and markedly corrugated, is uniform and thin in early fetal life, gradually becoming thicker with increasing gestational age [25]. The autofluorescence microscopy observation of the abdominal aorta wall of one IUGR stillbirth with higher aIMT revealed condensation of the elastic fibers in an evident and marked IEM. Fragmentation and partial duplication of the IEM was also evident, contrary to AGA fetuses. The study also highlighted the presence of inflammation elements within the arterial walls, such as CD68, a widely used marker for macrophages, usually absent in normal vessels. Molecules of the inflammatory cascade are known to be involved at every stage of the atherosclerotic process, from lesion initiation to plaque formation and rupture. Immune cells and their mediators, such as cytokines, soluble regulation factors, directly contribute to the chronic arterial inflammation that is a hallmark of atherosclerosis. Moreover, in the IUGR fetus positivity for E-selectin, a marker of activated EC was evident. Positivity for CD31, a marker for quiescent EC, was observed. All these data suggested that in IUGR fetus yhe endothelial cells in the intima were activated, although by unknown stimuli [30]. An observed positivity for beta actin, which indicated the presence of cells expressing non-muscle cytoskeletal actins usually absent in the arterial walls of second-trimester fetuses, was finally observed in IUGR stillbirth abdominal aorta wall, such as in pre-atherosclerotic lesions, representing de-differentiating smooth muscle cells, as a fibroblastoid phenotype [30].

When Skilton et al. [27] describe the measurement of abdominal aIMT in IUGR neonates, Cosmi et al. confirmed that aIMT measurements in IUGR fetuses and infants are inversely related to EFW, showing that low birth weight (LBW) and UA Doppler abnormalities may be correlated with an altered vascular structure causing possible endothelial damage, as described [31,32]. An increase in the aortic pulsatility index could be indicative of an increased arterial stiffness that might be similar to major environmental risk factors such as hypertension in childhood and adult life. These results have been confirmed in twin pregnancies, in which aIMT was greater in twins with IUGR and Doppler abnormalities, irrespective of sex and chorionicity [33].

Given the associations between aIMT and blood pressure (BP), as well as between birth size and offspring BP, the effects of prenatal factors on offspring BP may be partially explained by persistent aortic wall thickness in IUGR children [27, 31-33].

aIMT might not only identify the onset of IUGR and a class of fetuses at risk of cardiovascular remodelling as early as possible in utero, but also follow up patients in childhood in association to blood pressure measurements, to manage and improve their cardiovascular health condition, also controlling and modifying their life style during the time.

\section{Omics}

Although it is well established that the origins of IUGR are in early pregnancy and that the placenta plays an integral role in pregnancy outcome, the exact aetiologies of these multifactorial diseases remain poorly defined. Decades of research have not translated into a clear understanding of the underlying patho-physiologies or effective identification of women who are at high risk of developing IUGR.

Today there is wide consensus that information about the cellular and molecular biology of a process obtained by a comprehensive analysis of the protein component of a cell or tissue type significantly complements genomic data. Proteins regulate cellular actions and alterations in their expression are intrinsic to the understanding of any biological processes [34].

Although the interactions between the different 'omes' (genome, proteome, metabolome,) mean that omic strategies are complementary, there are differences in terms of the molecular properties of the analytes they cover (proteins and peptides, low molecular weight compounds, DNA oligomers) and therefore, the technologies required for preparation and analysis ${ }^{34}$. Proteomic methodologies are suitable for the detection of post-translational modifications such as phosphorylations and glycosylations that are linked to signaling processes. Parallel profiling of cellular proteins therefore, has great potential in unravelling signal transduction pathways [35]. In what is referred to as the "systems biology approach," all known genomic and proteomic data are to be combined with simulated mathematical models to study the cross-talk between molecular pathways and comprehend its regulation [36]. Metabolomics is defined as the quantitative measurement of the dynamic metabolic response of living systems to genetic, physical, pathological or developmental factors. The technology aimed at studying the metabolome(s) has emerged in numerous fields of research, including fetal medicine aiming to 
facilitate the understanding of fetal disease pathophysiology and discovery of predictive biomarkers [37].

Proteomic strategies have been used to investigate early pregnancy and trophoblast responses to altered oxygen tensions. In IUGR models proteomic and metabolomics investigations have demonstrated that several metabolites also differed in the culture medium from placental explants cultured in $1 \%$ compared with $6 \%$ and $20 \% \mathrm{O}_{2}$ [38].

IUGR fetuses present an early endothelial dysfunction represented by Doppler abnormalities and thicker aIMT occurring in utero that might play an important role in premature stiffening of the aortic vessels, in hypertension in childhood and in CVD in adult life [27,31]. Comparative proteome and metabolome profiling of normal and diseased cells contributed significantly to our understanding of the pathophysiologies of IUGR, translating into identification of disease markers and monitoring of disease progression.

Proteome analyses of cord blood serum and amniotic fluid samples of IUGR fetuses with a thicker aIMT have shown that IUGR condition alters the expression of proteins involved in the coagulation process, immune mechanisms, blood pressure and iron and copper homeostasis control [39] (Figure 2). A subsequent metabolomic approach in singleton IUGR fetuses have shown metabolomic alterations in cord blood and urine samples of restricted neonates [40]. The study highlights that IUGR fetuses can be accurately discriminated according to the ionic species detected through LC-HRMS analysis of serum metabolites (Figure 3). In particular an up-regulation of the amino acids phenylalanine, tryptophan, and glutamate was evidenced, suggesting a role of placental transport and metabolism. The same investigation was applied to twin pregnancies [41]. Thought to represent a special category of stunted fetal growth, twins have a higher risk of diseases associated with LBW and major incidence of morbidity and mortality [42]. Compared with singletons, the growth rate of twin fetuses is often characterized by a higher incidence of IUGR in one of the fetuses, varying from $15 \%$ to $25 \%$ in all monochorionic pregnancies, and frequently associated with Doppler velocimetry alterations [43].

In this investigation, a trend for increase of phenylalanine and a trend for down-regulation of valine, isoleucine, tryptophan, and proline were found in selective IUGR (sIUGR) twin fetuses with UA Doppler abnormalities compared with control co-twins. Moreover, a trend for increase of sphingosine was found in sIUGR twin with Doppler alterations (Figures 4 and 5). Sphingosine is an aliphatic amino alcohol component of all sphingoglycolipids, most of which are localized on the cell surface and are involved as second messengers in a variety of cellular signaling pathways such as for cellular growth, differentiation, and migration [44]. Sphingosine 1-phosphate, generated from sphingomyelin, is also expressed in the cardiovascular system and may be involved in the pathophysiology of diseases associated with endothelial dysfunction, such as in hypertension.

\section{Conclusion}

IUGR is a very complex and multifactorial disorder with long-term persistence of CVD later in patients who suffered IUGR early in life. In view of the ever-increasing prevalence of CVD in the developed and developing world, it will be important to identify and investigate mechanisms of early, potentially reversible pre-atherosclerotic changes in the endothelium. To date, the most clearly defined and well understood early precursor of atherosclerosis is ED. Several cellular mechanisms and markers of ED that could potentially lead to the development of early detection and therapeutic interventions have been determined.

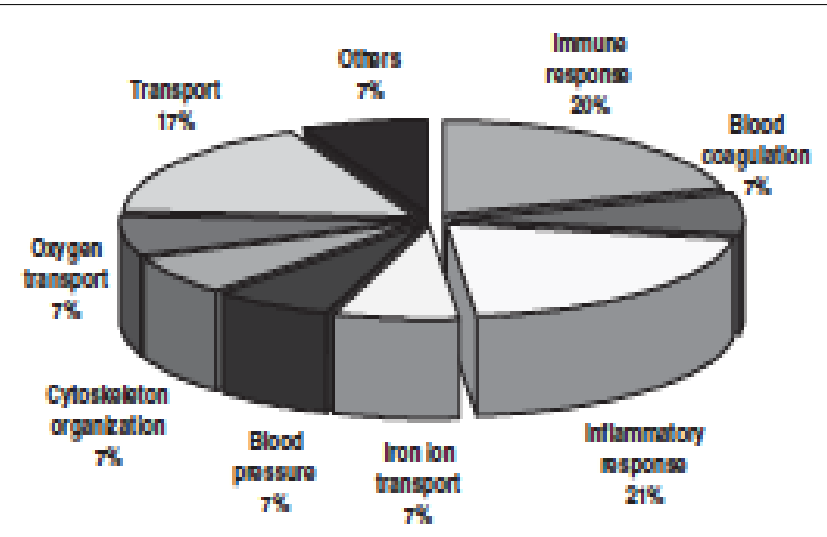

Figure 2: Protein GO categorisation of proteins differentially expressed in IUGR vs adequate for gestational age (AGA) fetuses. Distribution of the identified proteins according to the biological process in which they are involved. Go analysis was performed by using GeneCards database.

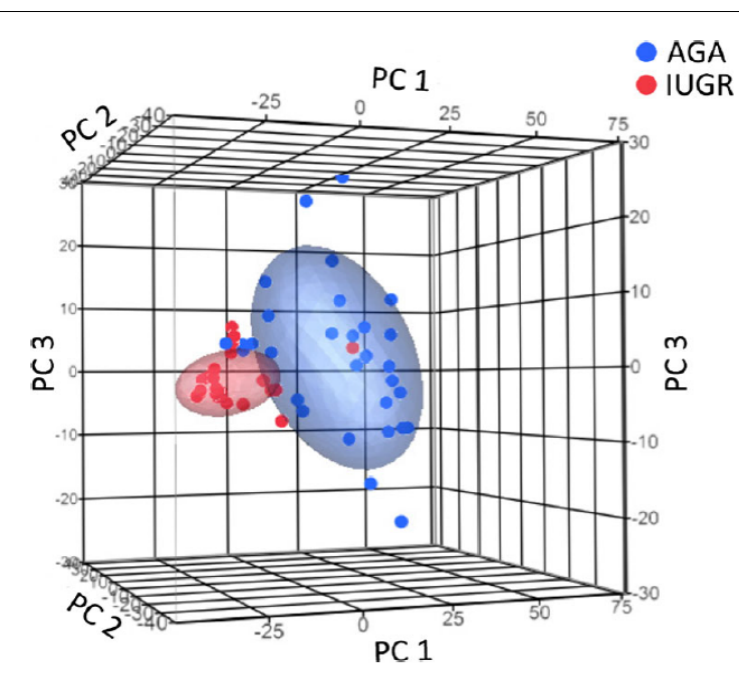

Figure 3: $3 D$ scatterplot of the first three principal components in principal component analysis (PCA) conducted on all the ionic species. The red ellipsoids are the apstial surfaces covering $50 \%$ of IUGR (blue) or adequate for gestational age (AGA, red) patients, respectively. The percentage of the first three PCs responsible for the observed separation was, respectively: PC1, 54\%, PC2, 10\% and PC3, $8 \%$.

\section{Ionic Species Identified in the LC-HRMS Spectra}

\begin{tabular}{lll}
\hline Metabolites & $\begin{array}{l}\text { Elemental } \\
\text { composition }\end{array}$ & $\begin{array}{l}\text { Label, according } \\
\text { to XCMS output }\end{array}$ \\
\hline Valine & $\mathrm{C}_{5} \mathrm{H}_{11} \mathrm{NO}_{2}$ & M118T101 \\
Tryptophan & $\mathrm{C}_{11} \mathrm{H}_{12} \mathrm{~N}_{2} \mathrm{O}_{2}$ & M205T241 \\
Proline & $\mathrm{C}_{5} \mathrm{H}_{9} \mathrm{NO}_{2}$ & M116T100 \\
Isoleucine & $\mathrm{C}_{6} \mathrm{H}_{13} \mathrm{NO}_{2}$ & M132T103 \\
Phenylalanine & $\mathrm{C}_{9} \mathrm{H}_{11} \mathrm{NO}_{2}$ & M166T141 \\
Choline & $\mathrm{C}_{5} \mathrm{H}_{13} \mathrm{NO}_{2}$ & M104T79 \\
Glycerophosphocholine & $\mathrm{C}_{26} \mathrm{H}_{52} \mathrm{NO}_{7} \mathrm{P}$ & M522T2096 \\
& & M544T2098 \\
& & M520T2032 \\
& & M518T2071 \\
& & M544T2032 \\
Sphingosine & & M396T2071 \\
& &
\end{tabular}

Figure 4: lonic species identified in the LC-HRMS Spectra in twin monochorionic diamniotic pregnancies complicated by selective intrauterine growth restriction. 
Citation: Favretto D, Cosmi E, Visentin S (2015) Application of Intima Media Thickness Measurement and Omics in Intra-uterine Growth Restriction Disease. Cardiol Pharmacol 4: 126. doi:10.4172/2329-6607.1000126

Page 5 of 6

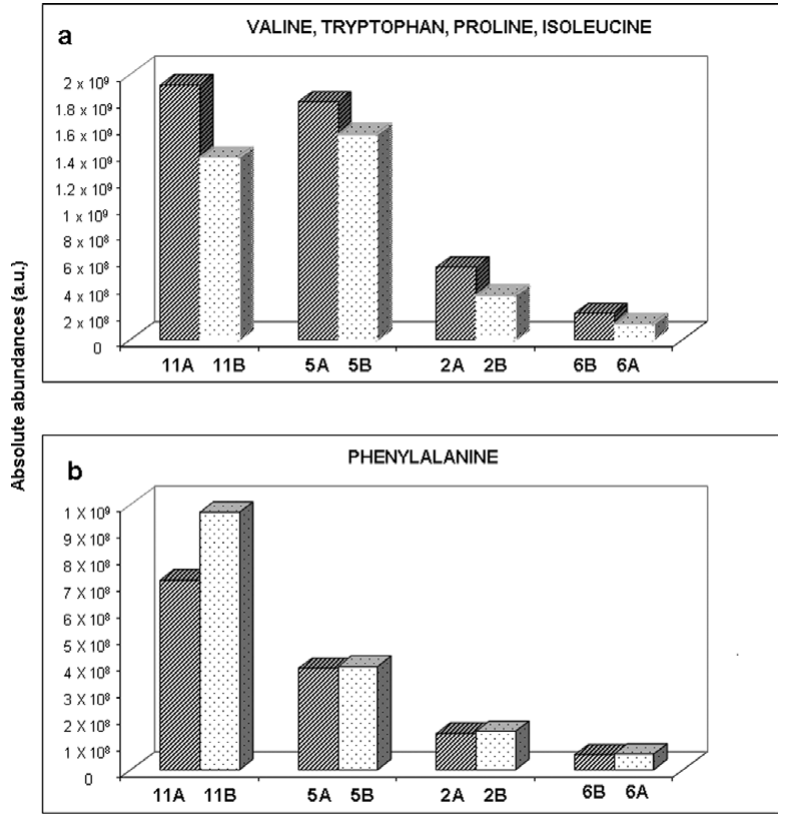

pregnancies

Figure 5: A comparison of the serum levels of amino acids valine, tryptophan, proline, isoleucine (a), and phenylalanine (b) between four selective IUGR (sIUGR) neonates with abnormal Doppler (dotted bars) and four appropriate for gestational age (AGA) controls (striped bars). Each subject was considered as an independent statistical unit.

'Omic' technologies represent a strategy towards high-throughput, simultaneous analysis of thousands of biological molecules. Their development has been accelerated in the post-genomic era since these molecules represent the interaction of genes and environment or the 'functional genome'. Omics domains are of particular interest in the search for predictive disease biomarkers and have additional relevance in understanding pathophysiology and the development of molecularly targeted therapeutics.

Innovative interdisciplinary research in the areas of nutrition, reproductive physiology, and vascular biology will play an important role in developing new strategies of intervention. Future pharmaceutical therapies and innovative alternative nutritional/environmental strategies even could maximize population-based wellbeing [45].

\section{References}

1. Committee on Practice Bulletins-Obstetrics, American College of Obstetricians and Gynecologists.

2. American College of Obstetricians and Gynecologists (2013) ACOG Practice bulletin no. 134: fetal growth restriction. Obstet Gynecol 121: 1122-1133.

3. Barker DJ (2004) The developmental origins of well-being. Philos Trans R Soc Lond B Biol Sci 359: 1359-1366.

4. Barker DJ (2004) The developmental origins of well-being. Philos Trans R Soc Lond B Biol Sci 359: 1359-1366.

5. Gardosi J, Mongelli M, Wilcox M, Chang A (1995) An adjustable fetal weight standard. Ultrasound Obstet Gynecol 6: 168-174.

6. Gardosi J, Mongelli M, Wilcox M, Chang A (1995) An adjustable fetal weight standard. Ultrasound Obstet Gynecol 6: 168-174.

7. Royal College of obstetricians and Gynecologists (2002) The investigation and management of the small for gestational age fetus. Guideline No. 31. Technical report, Royal College of obstetricians and gynaecologist, London, UK.
8. Hernandez-Andrade E, Serralde JA, Cruz-Martinez R (2012) Can anomalies of fetal brain circulation be useful in the management of growth restricted fetuses? Prenat Diagn 32: 103-112.

9. Bernstein IM, Horbar JD, Badger GJ, Ohlsson A, Golan A (2000) Morbidity and mortality among very-low-birth-weight neonates with intrauterine growth restriction. The Vermont Oxford Network. Am J Obstet Gynecol 182: 198-206.

10. Gardosi J, Madurasinghe V, Williams M, Malik A, Francis A (2013) Maternal and fetal risk factors for stillbirth: population based study. BMJ 346: f108.

11. Baschat AA, Weiner CP (2000) Umbilical artery doppler screening for detection of the small fetus in need of antepartum surveillance. Am J Obstet Gynecol 182: $154-158$.

12. Bilardo CM, Wolf H, Stigter RH, Ville Y, Baez E, et al. (2004) Relationship between monitoring parameters and perinatal outcome in severe, early intrauterine growth restriction. Ultrasound Obstet Gynecol 23:119-25.

13. Rizzo G, Capponi A, Cavicchioni O, Vendola M, Arduini D (2008) Low cardiac output to the placenta: an early hemodynamic adaptive mechanism in intrauterine growth restriction. Ultrasound Obstet Gynecol 32: 155-159.

14. White CR, Haidekker M, Bao X, Frangos JA (2001) Temporal gradients in shear but not spatial gradients, stimulate endothelial cell proliferation. Circulation 103: 2508-2513.

15. Li G, Xiao Y, Estrella JL, Ducsay CA, Gilbert RD, et al. (2003) Effect of fetal hypoxia on heart susceptibility to ischemia and reperfusion injury in the adult rat. J Soc Gynecol Investig 10: 265-274.

16. Baum M (2010) Role of the kidney in the prenatal and early postnatal programming of hypertension. Am J Physiol Renal Physiol 298: F235-247.

17. Barker DJ, Eriksson JG, Forsén T, Osmond C (2002) Fetal origins of adult disease: strength of effects and biological basis. Int J Epidemiol 31: 1235-1239.

18. World Health Organization (2009) Global health risks: Mortality and burden of disease attributable to selected major risks. Geneva: WHO.

19. Morganti M, Carpi A, Nicolini A, Gorini I, Glaviano B, et al. (2002) Atherosclerosis and cancer: common pathways on the vascular endothelium. Biomed Pharmacother 56: 317-324.

20. Yang G, Lucas R, Caldwell R, Yao L, Romero MJ, et al. (2010) Nove mechanisms of endothelial dysfunction in diabetes. J Cardiovasc Dis Res 1 59-63.

21. Förstermann U, Münzel T (2006) Endothelial nitric oxide synthase in vascular disease: from marvel to menace. Circulation 113: 1708-1714.

22. Yokoyama M (2004) Oxidant stress and atherosclerosis. Curr Opin Pharmacol 4: $110-115$.

23. Kuzkaya N, Weissmann N, Harrison DG, Dikalov S (2003) Interactions of peroxynitrite, tetrahydrobiopterin, ascorbic acid, and thiols: implications for uncoupling endothelial nitric-oxide synthase. J Biol Chem 278: 22546-22554.

24. Libby P, Ridker PM, Maseri A (2002) Inflammation and atherosclerosis. Circulation 105: 1135-1143.

25. Osto E, Cosentino $F(2010)$ The role of oxidative stress in endothelial dysfunction and vascular inflammation. In: Ignarro LJ (ed) Nitric Oxide: Biology and Pathobiology (2nd edn) London: Academic Press: 705-754.

26. Blake GJ, Ridker PM (2001) Novel clinical markers of vascular wall inflammation Circ Res 89: 763-771.

27. McGill HC Jr, McMahan CA, Herderick EE, Malcom GT, Tracy RE, et al. (2000) Origin of atherosclerosis in childhood and adolescence. Am J Clin Nutr 72 1307S-1315S.

28. Shin PS, Kim DS (1963) Histochemical studies of fetal arteries of Koreans with special reference to atherogenesis in adults. Yonsei Med J 4: 37-42.

29. Münzel T, Sinning C, Post F, Warnholtz A, Schulz E (2008) Pathophysiology, diagnosis and prognostic implications of endothelial dysfunction. Ann Med 40 : 180-196.

30. Skilton MR, Evans N, Griffiths KA, Harmer JA, Celermajer DS (2005) Aortic wal thickness in newborns with intrauterine growth restriction. Lancet 365: 14841486.

31. Aggoun Y, Szezepanski I, Bonnet D (2005) Noninvasive assessment of arteria stiffness and risk of atherosclerotic events in children. Pediatr Res 58: 173-178. 
Citation: Favretto D, Cosmi E, Visentin S (2015) Application of Intima Media Thickness Measurement and Omics in Intra-uterine Growth Restriction Disease. Cardiol Pharmacol 4: 126. doi:10.4172/2329-6607.1000126

Page 6 of 6

32. Meyer WW, Lind J, Yao AC, Kauffman SL (1982) Early arterial lesions in infancy and childhood and ways of prevention. Paediatrician 11: 136-156.

33. Lo Vasco VR, Salmaso R, Zanardo V, Businaro R, Visentin S, et al. (2011) Fetal aorta wall inflammation in ultrasound-detected aortic intima/media thickness and growth retardation. J Reprod Immunol 91: 103-107.

34. Cosmi E, Visentin S, Fanelli T, Mautone AJ, Zanardo V (2009) Aortic intima media thickness in fetuses and children with intrauterine growth restriction. Obstet Gynecol 114: 1109-1114.

35. Järvisalo MJ, Jartti L, Näntö-Salonen K, Irjala K, Rönnemaa T, et al. (2001) Increased aortic intima-media thickness: a marker of preclinical atherosclerosis in high-risk children. Circulation 104: 2943-2947.

36. Visentin S, Grisan E, Zanardo V, Bertin M, Veronese E, et al. (2013) Developmental programming of cardiovascular risk in intrauterine growthrestricted twin fetuses according to aortic intima thickness. J Ultrasound Med 32: $279-284$

37. Banks R, Selby P (2003) Clinical proteomics--insights into pathologies and benefits for patients. Lancet 362: 415-416.

38. Phizicky E, Bastiaens PI, Zhu H, Snyder M, Fields S (2003) Protein analysis on a proteomic scale. Nature 422: 208-215.

39. Ideker T, Galitski T, Hood L (2001) A new approach to decoding life: systems biology. Annu Rev Genomics Hum Genet 2: 343-372.
40. Nicholson JK, Lindon JC, Holmes E (1999) "Metabonomics": understanding the metabolic responses of living systems to pathophysiological stimul via multivariate statistical analysis of biological NMR spectroscopic data. Xenobiotica 29:1181-1189.

41. Heazell A, Brown M, Dunn W, Worton S, Crocker IP, et al. (2008) Analysis of the metabolic footprint and tissue metabolome of placental villous explants cultured at different oxygen tensions reveals novel redox biomarkers. Placenta 29:691-698.

42. Cecconi D, Lonardoni F, Favretto D, Cosmi E, Tucci M, et al. (2011) Changes in amniotic fluid and umbilical cord serum proteomic profiles of foetuses with intrauterine growth retardation. Electrophoresis 32: 3630-3637.

43. Favretto D, Cosmi E, Ragazzi E, Visentin S, Tucci M, et al. (2012) Cord blood metabolomic profiling in intrauterine growth restriction. Anal Bioanal Chem 402: 1109-1121.

44. Cosmi E, Visentin S, Favretto D, Tucci M, Ragazzi E, et al. (2013) Selective intrauterine growth restriction in monochorionic twin pregnancies: markers of endothelial damage and metabolomic profile. Twin Res Hum Genet 16: 816826

45. Blickstein I, Kalish RB (2003) Birthweight discordance in multiple pregnancy. Twin Res 6: 526-531. 